\title{
Internet of Robotics Things (IoRT) Based Integration of Robotic Applications for Advanced Research
}

\author{
https://doi.org/10.31185/wjcm.Vol1.Iss1.4 \\ Asst.Prof.Dr. HM Verma $(\bowtie)$ \\ Computer Science Dept.,ITM University, Gwalior,India \\ hmvermadituniversity.ac.in
}

\begin{abstract}
IoT and robotics industries are united to create the Internet of Robotics Things (IoRT). IoRT is the idea of intelligent machines monitoring the environment around them and using local and distributed intelligence to decide on courses of action and making decisions accordingly. IOT is a network of devices that are connected to the internet, including devices and equipment connected by sensors. These elements are essential for businesses trying to drive customer facing innovation, data-driven decisions, new applications, digital transformation, business models and revenue streams. Robots need to maintain great flexibility to react to unexpected conditions. AI helps these robots to deal with any unforeseen circumstances. Robotics and Simulation are key elements in the solutions of advancing manufacturing and production. Many people often think of IoT and robotics technologies in separate fields but they have been getting increasingly close in recent years. The In this way, such processes are used to avoid the loss of human life and the automation of processes which need high performances. Robotic and simulated application have been successfully deployed to functions in real world scenarios that man will not be able to accomplish such as study of volcanoes, and space center on its own. Furthermore, the robotic implementations give robots the opportunity to efficiently and safely function in the adverse conditions without being injured physically.
\end{abstract}

Keywords - Internet of Things, Internet of Robotic Things, IoT integrated Robotics, Robots in IoT Environment

\section{Introduction}

Internet of Things (IoT) devices and robots rely on sensors to gather information about their surroundings, analyze this information and make decisions accordingly. Robots are more qualified to deal with unforeseen circumstances, while IoT applications are limited to handling well-defined tasks. The main distinction between the robotics community and the IoT is that robots are active and operate in the real world. It happens. Besides, focus has shifted from the cyber component of the Internet of Things to the physical one and that's where the efforts are focused now.

Thus far, the robotics and IoT communities have been somewhat dominated by various yet closely related objectives. IOT focuses on supporting services, while robotic 
communities focus on production action, interaction and autonomy. By combining two ideas, the value would be increased.

This was just how Internet of Robotic Things was conceived. Enhanced situational awareness allows robots to accomplish their task more effectively in the IoT sensor and data analytics technologies.

Automation is not a new idea, but it is becoming more popular with the rise in labor costs. By using robots in the workplace, we can boost overall productivity with the same number of workers.

As for IoT applications, they are able for both mobile and stationary applications. Some would stick to the program while others would change the program. The more sophisticated sensors that these robots have will become an important selection for both customers and manufacturers.

A number of free and open source robotic platforms and simulation libraries are available which can be used by the scientists to automate their work to fetch the predicted results. Once the results on simulations and robotic platforms are found suitable, then further actions are planned [1,2].

The Robotic platforms provide the set of software, databases and conventions that seek to simplify the challenge of developing complex and robust robot actions across a wide range of real time industrial situations. From the robot's view, challenges that appear simple to humans also differ wildly between instances of activities and conditions. The sensitive environment where automated machines or programmable devices can be placed, such robotic systems help a lot. The scientists and data analysts can fetch the data via satellite or assorted wireless protocols while sitting at remote control room [3, 4].

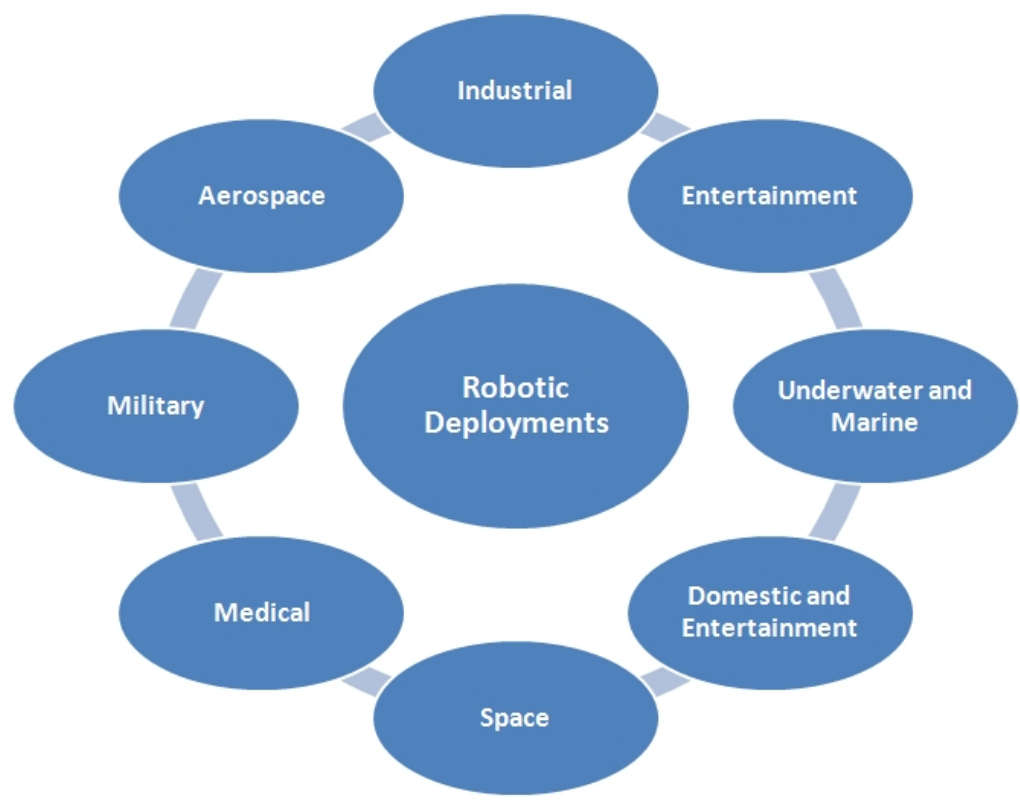

Fig. 1. Key Dimensions of Robotic Deployments 
The typical features of robotic platforms $[5,6]$ include

- Importing the templates from CAD for mechanical and production engineers

- Integration of OpenStreeMap and Google Maps for real time locations

- $\quad$ Easy interface to work

- $\quad$ Plugins and icons for different automations including automobile, space, drones, aerospace vehicles, volcano, underwater, marine, aircrafts and many others

- Integration with multiple programming languages for customization of features

- $\quad$ Exporting the results in multiple formats

- Association with multiple libraries of different engineering domains for compatibility

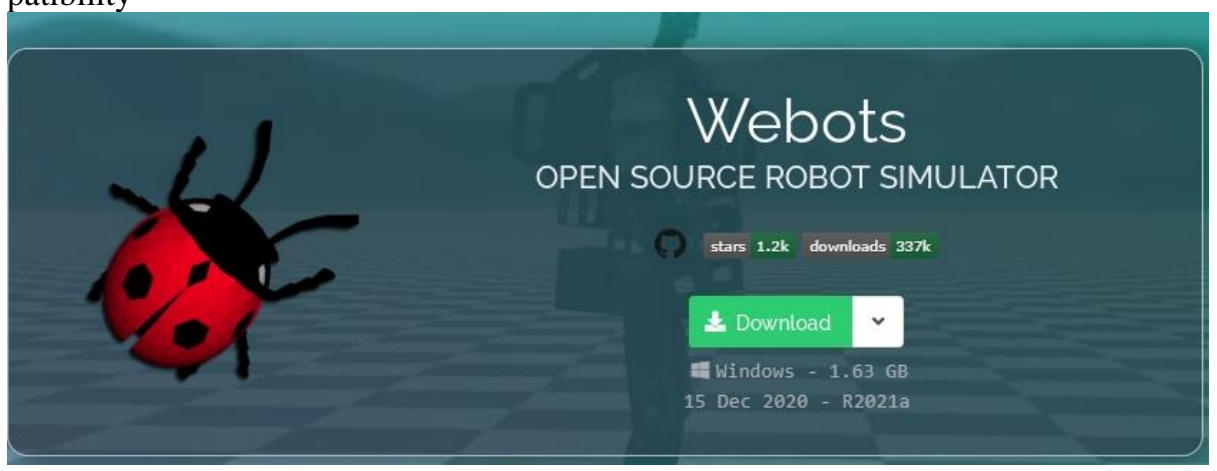

Fig. 2. Official Portal of Webots

Following are the free and open-source robotics development frameworks which are widely used for industrial, social, corporate, military and related applications. These are programmable libraries with support to customize as per the requirements of the domain in which to implement [7, 8, 9].

Table 1. Free and Open Source Platforms for Robotic Simulations

\begin{tabular}{|l|l|}
\hline Robotic Simulation Platform & URL \\
\hline Webots & cyberbotics.com \\
\hline Gazebo & gazebosim.org \\
\hline Robot Operating System & ros.org \\
\hline Open Robot Control Software & orocos.org \\
\hline Yet Another Robot Platform & yarp.it/git-master \\
\hline Mobile Robot Programming Toolkit & mrpt.org \\
\hline Robatarium & robotarium.gatech.edu \\
\hline Poppy-Project & poppy-project.org \\
\hline CoppeliaSim & coppeliarobotics.com \\
\hline
\end{tabular}




\section{Working with Robotic Scenarios for Industrial and Corporate Applications}

CoppeliaSim is a cross platform tool for simulation and industrial robotic applications. This tool makes possible to create the portable, scalable, and easy-to-maintain simulations for multiple scenarios [10,11, 12].

This framework is having interfaces to various programming languages like $\mathrm{C}$, Lua, Java, Python, Matlab and Octave. For an interactive production environment, the CoppeliaSim Robot Simulator relies on a distributed control architectural system in which the individual control of each object or model requires an embedded script for high performance control based applications. Such features render CoppeliaSim with extremely versatile and adaptable powers for broad range of robotic systems [13].

CoppeliaSim is used in the development of high-speed algorithms, plant modeling, rapid prototyping, validations, remote control, double-check security, automated double-checking and many others $[14,15]$.

Following are the key features in CoppeliaSim which makes this platform highly effective and performance based for multiple applications including engineering, military, aerospace, health sciences and many others $[16,17]$

- $\quad$ Remote APIs

- Collision Detection

- Minimum Distance Calculation

- Dynamics / Physics

- Dynamic Particles

- Kinematics

- $\quad$ Proximity Sensor Simulation

- Vision Sensor Simulation

- $\quad$ Data Recording \& Visualization

- $\quad$ Building Block Concept

- $\quad$ Path and Motion Planning

- Integrated Editing Modes for Customization

- $\quad$ Custom User Interfaces

- Convenient and Effective Model Browser

- $\quad$ High Performance Data Import / Export

- $\quad$ RRS Interface \& Motion Library

- $\quad$ Full Interaction Modes in Multiple Dimensions and Coordinates

- $\quad$ Full-Featured Scene Hierarchy

The implementations including the convergence of dynamics and physics can be achieved using CoppeliaSim with Bullet mechanics, Newton, Differential Equations and other integrations for easy and effective dynamic calculations simulating real life physical and entity encounters which include collision reaction, grabbing, collision response, grasping and many others. Multiple recordable data sources (including userdependent) can view or combine time graphs with xy-graphs or 3D curves can be achieved effectively $[18,19,20]$. 
CoppeliaSim integrates features for real time automations including next to proximity sensors, vision sensors, viewable objects for dynamic environment with camera objects [21].

\section{Collision Detection and Avoidance in CoppeliaSim}

Following the brief description of implementation associated with the collision detection and avoidance that is one of the key features in robots. For industrial and corporate applications, if a robot is developed, that robot should be programmed in such a way that it should not collide to other components or machinery in the industrial unit.

The collision detection and avoidance module is easy to program in CoppeliaSim so that the look-and-feel of the robotic application will be there. In Menu->Tools->Calculation the distance and collision settings can be set up effectively.
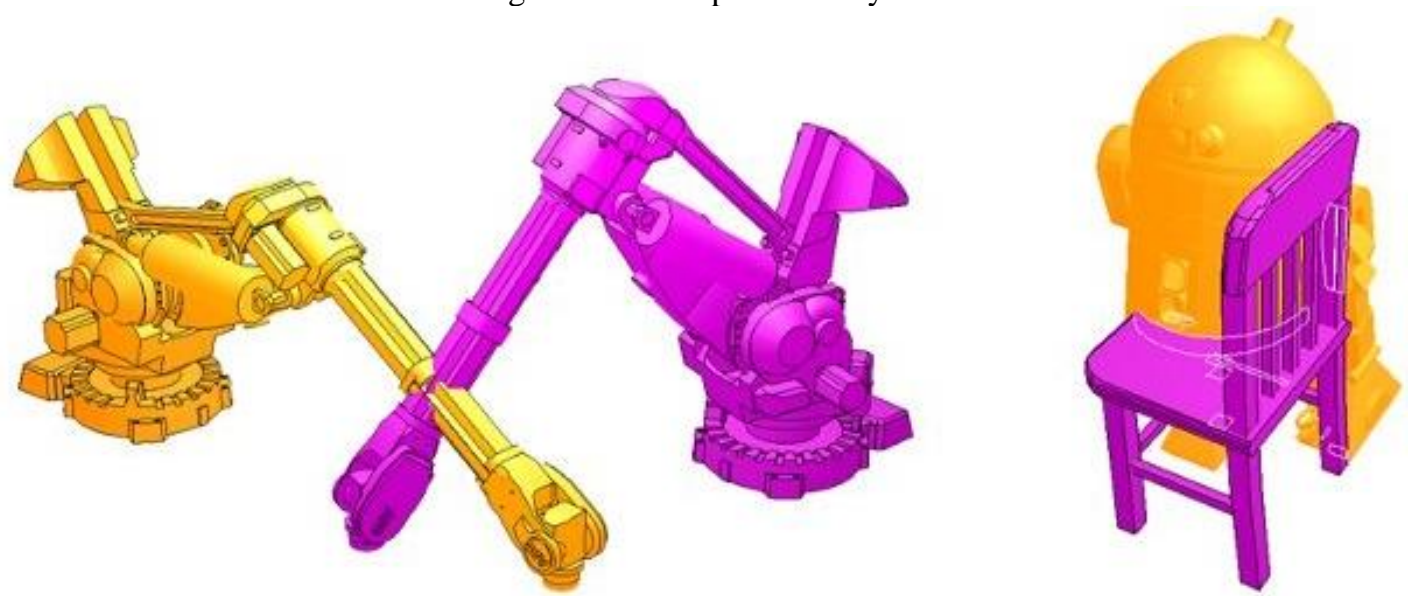

Fig. 3. Collision Detection in Robotic Platform of Coppelia 


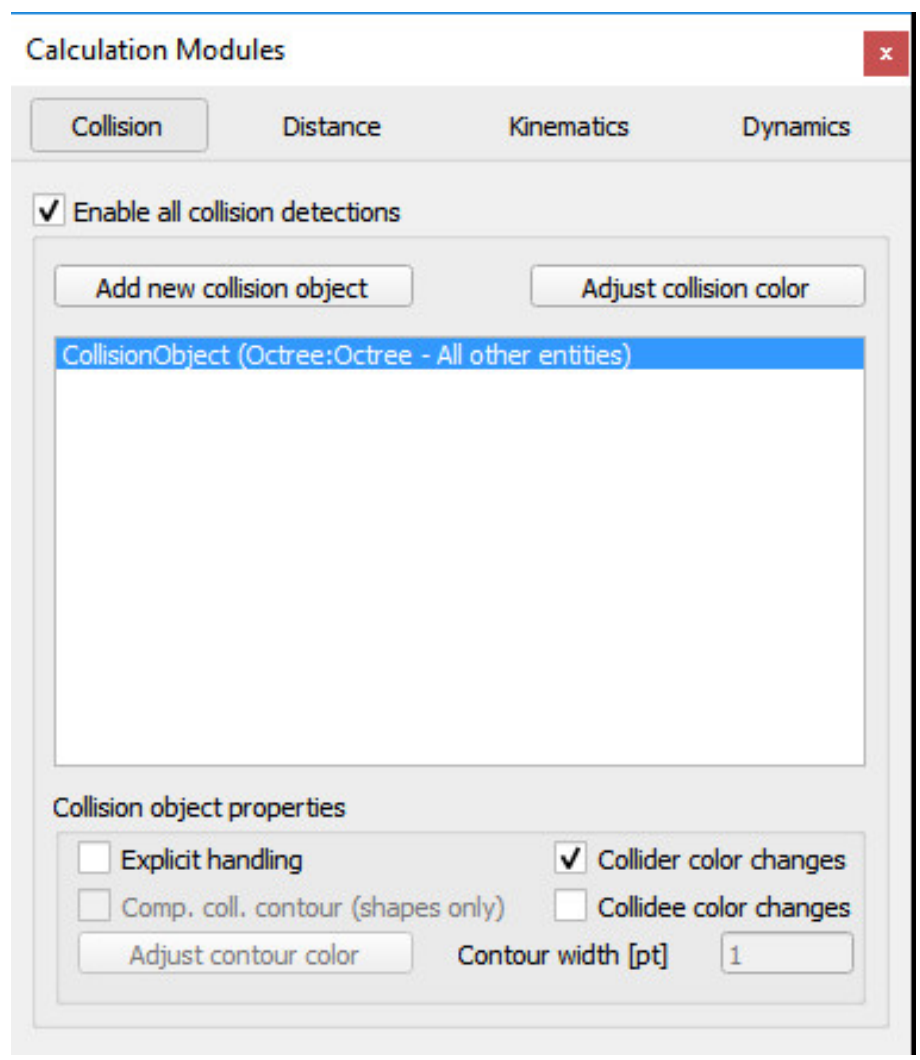

Fig. 4. Setting Panel to Detect Distance for Collection Avoidance

\section{Conclusion}

The scientists and practitioners are having huge scope to simulate their research tasks using these open source platforms for robotic applications where actual infrastructure and devices are very costly to implement. Rather than using the actual hardware and gadgets, the open source libraries and frameworks provide the ease to researchers to create, simulate and fetch the research based outcomes from their algorithms and imaginations.

\section{References}

[1] Ahmadi H, Arji G, Shahmoradi L et al (2018) The application of internet of things in healthcare: a systematic literature review and classification. Univ Access Inf Soc. https://doi.org/10.1007/s10209-018-0618-4 
[2] Albahri AS, Zaidan AA, Albahri OS et al (2018) Real-time fault-tolerant mhealth system: comprehensive review of healthcare services, opens issues, challenges and methodological aspects. J Med Syst 42:137. https://doi.org/10.1007/s10916-018-0983-9

[3] Ann OC, Theng LB (2014) Human activity recognition: a review. In: IEEE international conference on control system, computing and engineering (ICCSCE 2014), pp 389-393. https://doi.org/10.1109/ICCSCE.2014.7072750

[4] Bal M, Shen W, Hao Q et al (2011) Collaborative smart home technologies for senior independent living: a review. In: 15th international conference on computer supported cooperative work in design (CSCWD), pp 481-488. https://doi.org/10.1109/CSCWD.2011.5960116

[5] Bogue R (2013) Robots to aid the disabled and the elderly. Ind Robot

[6] Brownsell S, Hawley M (2004) Fall detectors: do they work or reduce the fear of falling? Hous Care Support 7:18-24

[7] Buttorff C, Ruder T, Bauman M (2017) Multiple chronic conditions in the United States. Santa Monica

[8] Camarinha-Matos LM, Ferrada F, Oliveira AI et al (2014) Care services provision in ambient assisted living. IRBM 35:286-298

[9] Celler BG, Hesketh T, Earnshaw W et al (1994) An instrumentation system for the remote monitoring of changes in functional health status of the elderly at home. In: 16th annual international conference of the IEEE engineering in medicine and biology society, IEEE, pp 908-909

[10] Centers for Medicare and Medicaids Services (2018) NHE Fact Sheet 2017. US Centers for Medicare and Medicaid Services. https://www.cms.gov/research-statistics-data-and-systems/statistics-trends-and-reports/nationalhealthexpenddata/nhe-fact-sheet.html. Accessed 10 Jan 2019

[11] Chan M, Esteve D, Escriba C et al (2008) A review of smart homes - present state and future challenges. Comput Methods Programs Biomed 91:55-81. https://doi.org/10.1016/j.cmpb.2008.02.001

[12] Chen S, Lach J, Lo B et al (2016) Toward pervasive gait analysis with wearable sensors: a systematic review. IEEE J Biomed Health Informatics 20:1521-1537. https://doi.org/10.1109/JBHI.2016.2608720

[13] Chiarini G, Ray P, Akter S et al (2013) mHealth technologies for chronic diseases and elders: a systematic review. IEEE J Sel Areas Commun 31:6-18. https://doi.org/10.1109/JSAC.2013.SUP.0513001

[14] Coradeschi S, Kristoffersson A, Loutfi A et al (2011) Towards a methodology for longitudinal evaluation of social robotic telepresence for elderly. In: Human robot interaction, 2011

[15] Dakurah MN, Koo C, Choi W et al (2015) Implantable bladder sensors: a methodological review. Int Neurourol J 19:133-141. https://doi.org/10.5213/inj.2015.19.3.133

[16] Elena-Lenz C (2014) Internet of things: six key characteristics. https://designmind.frogdesign.com/2014/08/internet-things-six-key-characteristics/. Accessed 10 Sept 2019

[17] Grant MJ, Booth A (2009) A typology of reviews: an analysis of 14 review types and associated methodologies. Health Inf Libr J 26:91-108. https://doi.org/10.1111/j.14711842.2009.00848.x

[18] Jaul E, Menzel J (2014) Pressure ulcers in the elderly, as a public health problem. J General Pract 2:174. https://doi.org/10.4172/2329-9126.1000174

[19] Kalid N, Zaidan AA, Zaidan BB et al (2017) Based-real time remote health monitoring systems: a review on patients prioritization and related "big data" using body sensors information and communication technology. J Med Syst 42:30. https://doi.org/10.1007/s10916017-0883-4 
[20] Khosravi P, Ghapanchi AH (2016) Investigating the effectiveness of technologies applied to assist seniors: a systematic literature review. Int J Med Informatics 85:17-26. https://doi.org/10.1016/j.ijmedinf.2015.05.014

[21] Klimova B (2016) Mobile health devices for aging population groups: a review study. In: Younas M, Awan I, Kryvinska N, Strauss C, Thanh DV (eds) Mobile web and intelligent information systems. Springer International Publishing, Cham, pp 295-301

Article submitted 16 January 2021. Published as resubmitted by the authors 1 April 2021. 